BULLETIN Bulletin hispanique

HISPANIQUE Université Michel de Montaigne Bordeaux

115-1 | 2013

Poésie et société en Espagne : 1650-1750

\title{
Unidades fraseológicas y TIC
}

Instituto Cervantes, 2012

\section{Alexandra Oddo}

\section{(2) OpenEdition}

Journals

Édition électronique

URL : http://journals.openedition.org/bulletinhispanique/2586

DOI : 10.4000/bulletinhispanique.2586

ISSN : 1775-3821

Éditeur

Presses universitaires de Bordeaux

Édition imprimée

Date de publication : 1 juin 2013

Pagination : $377-379$

ISBN : 978-2-86781-898-1

ISSN : 0007-4640

Référence électronique

Alexandra Oddo, "Unidades fraseológicas y TIC », Bulletin hispanique [En ligne], 115-1 | 2013, mis en ligne le 08 décembre 2013, consulté le 23 septembre 2020. URL : http://journals.openedition.org/ bulletinhispanique/2586 ; DOI : https://doi.org/10.4000/bulletinhispanique.2586

Ce document a été généré automatiquement le 23 septembre 2020.

Tous droits réservés 


\title{
Unidades fraseológicas y TIC
}

Instituto Cervantes, 2012

\author{
Alexandra Oddo
}

\section{RÉFÉRENCE}

Unidades fraseológicas y TIC, edición a cargo de María Isabel González Rey, Biblioteca

fraseológica y paremiológica, Serie «Monografías», n² 2, CVC, Instituto Cervantes, 2012, $307 \mathrm{p}$.

1 Les travaux publiés par les groupes de recherches espagnols sur la parémiologie et la phraséologie connaissent un vif succès, dynamisé par une production régulière et féconde. Ce numéro monographique de la collection Biblioteca fraseológica y paremiológica de l'Institut Cervantès est le fruit d'un travail collectif dirigé par $\mathrm{M}^{\mathrm{a}}$. I. Gónzalez Rey et intégralement consacré aux apports des nouvelles technologies de l'information et de la communication à l'étude des unités phraséologiques. Le volume répond à un besoin réel dans ce domaine de spécialité en pleine expansion : évoquons simplement à ce sujet les créations récentes de bases de données, les nouveaux outils de la recherche lexicographique et phraséographique, le développement de l'outil informatique. Le volume est en ce sens d'une grande rigueur et d'une grande fiabilité dans la présentation des dernières avancées de la recherche dans ces domaines et offre aux lecteurs un panorama précis des différentes directions envisagées par les parémiologues et les phraséologues espagnols: il signale les différentes bases de données qui s'organisent autour des unités phraséologiques dans divers sites espagnols (Madrid, Barcelone, Santiago, Alicante, entre autres) et s'attache à en expliquer à la fois le fonctionnement, les ressources et le potentiel d'exploitation mais aussi les objectifs et les champs disciplinaires concernés. Et les enjeux sont clairement établis: l'exploitation de ces données embrasse l'ensemble des domaines variés des sciences humaines, la langue, la traduction, l'enseignement, par exemple, et à un autre niveau, la conservation d'un patrimoine linguistique et culturel. 
Comme le rappelle avec beaucoup d'humour André Gallego Barnés, si ces nouvelles technologies représentent une véritable révolution dans un domaine qui se matérialise dans des collections colossales de parémies et de phrases figées, elles exigent aussi, pour leur manipulation, certaines compétences. Les premiers articles reviennent sur la création de corpus (BADARE= Base de datos sobre refranes del calendario y meteorológicos en la Romania présenté par José Enrique Gargallo Gil ; Le Refranero multilingüe, présenté par Julia Sevilla Muñoz; la base de données multilingues d'unités phraséologiques du groupe FRASYTRAM dirigé par Pedro Mogorrón Huerta, la base de données destinée à l'élaboration d'un dictionnaire phraséologique espagnol/arabe développée par Mona Salah Eldin Shalan, du Caire) et sur leur potentiel d'exploitation. Ils nous donnent à voir un nombre considérable d'applications pratiques dans les champs de l'enseignement, de la traduction (avec un article de Silvia Molina Plaza consacré au cas des collocations, p. 257-268 et une présentation de $\mathrm{M}^{\mathrm{a}}$ Lucía Navarro Brotons au sujet de la traduction des proverbes dans les textes journalistiques, p. 299-307), de la linguistique et notamment dans l'étude des variantes diatopiques (p. 29-50) et diastratiques (p. 63-82). De tels corpus doivent être envisagés comme des outils capables d'améliorer nos connaissances dans les domaines de la phraséologie et de la parémiologie, des outils susceptibles de régler certaines des grandes questions typologiques et taxinomiques inhérentes à ces disciplines.

3 Les recherches entreprises grâce aux TIC repoussent constamment les limites de nos connaissances dans ces domaines. Les théories du Lexique-Grammaire se poursuivent par exemple dans les travaux de Giovanna Angela Mura sur la recherche de critères permettant d'isoler les traits des schémas phraséologiques syntaxiques. Les corpus se spécialisent et livrent autant d'instantanés dans des domaines comme l'espagnol coloquial ou encore l'espagnol des adolescents et des jeunes adultes. Cette linguistique de corpus trouve tout naturellement une application dans l'approche contrastive des langues (italien/espagnol, p.97-109; allemand/espagnol, p.147-166, avec la présentation de Carmen Mellado Blanco) qui permet de revisiter les équivalences entre langues et de mieux saisir des phénomènes de polysémie, de synonymie ou d'antonymie.

4 La linguistique de corpus se doit d'envisager le Web comme un immense modèle ou, plus exactement, comme le corpus synchronique par excellence, à la fois témoignage de pratiques orales et écrites. Les travaux de Marina García Yelo, de Mario Marcon et de Carmen Mellado Blanco interrogent les réseaux sociaux et les moteurs de recherche (Facebook, Twitter, Google, CREA) afin d'étudier le taux de pénétration des proverbes dans les NTIC et d'en étudier les emplois (compétence parémiologique ; détournements, p.111-124; études de familiarité et études de fréquence, p.125-146). Finalement, les unités phraséologiques dont le sens polylexical est codé, trouvent dans les NTIC un formidable moyen de sortir du cantonnement des dictionnaires: dans la constitution de corpus de spécialité et de nouveaux supports ces lexies complexes se dotent des outils technologiques nécessaires au développement d'une véritable phraséographie.

Pour finir, le volume accorde une large place au volet didactique qui s'ouvre par l'intermédiaire d'une pratique croisée des NTIC et de la phraséologie/ parémiologie. Les articles de Germán Conde Tarrío et de Vanessa Fernández Pampín font le point sur les différentes exploitations du Refranero Multilingüe dans l'enseignement/apprentissage des langues étrangères ou régionales. $\mathrm{M}^{\mathrm{a}}$. Ángeles Solano Rodríguez, Justyna Pietrzak, Maria Antonella Sardelli et Natalia Vyshnia se penchent en particulier sur 
l'enseignement secondaire. Ces études montrent le lien étroit qui existe entre l'apprenant et les NTIC et soulignent l'évidente adéquation entre apprentissage et utilisation d'une technologie qui est au centre des intérêts de l'apprenant. Dans les domaines de la didactique et de l'enseignement, l'emploi des NTIC s'impose comme le garant d'une progression des compétences assurée par l'attrait de ses propres caractéristiques: la connectivité, l'interactivité et le multimédia. Maddalena Ghezzi, Lucía Serrano Lucas et Manuel Sevilla Muñoz évoquent, d'une façon plus générale, l'impact de ces nouvelles technologies sur l'enseignement spécifique de la traduction.

6 Pragmatique, le volume ne perd jamais de vue les applications pratiques des données théoriques qu'il fournit. Appréciée par le lecteur, la cohérence de sa construction est un atout qui permet de se faire une idée précise du potentiel mis à la disposition des phraséologues et des parémiologues pour la création de corpus, leur analyse et leur exploitation dans les diverses branches des sciences humaines.

\section{AUTEUR}

\section{ALEXANDRA ODDO}

Université Paris Ouest Nanterre La Défense - EA 369 Études Romanes 